\section{Is vision metric? Comment on Lappin and Love (1992)}

\section{ZYGMUNT PIZLO and MONIKA SALACH-GOLYSKA Purdue University, West Lafayette, Indiana}

In a recent paper, Lappin and Love (1992) showed that depth judgments, based on planar motion of a stimulus, are remarkably precise. This led them to the conclusion that perception of planar motion allows acquiring metric information about visual space. We show, however, that Lappin and Love's stimuli contained 2-D cues in the frontal plane that could subserve the subject's judgments. This means that high precision in this task does not necessarily imply metric structure of visual space.

There have recently been several studies relevant to the question of whether vision is metric. These studies examined precision and accuracy of judgments about depth. Most of them implied that vision does not allow the acquisition of metric information about 3-D space. Consider, first, the precision of depth judgments. Todd and colleagues (Todd \& Bressan, 1990; Todd \& Norman, 1991; Todd and Reichel, 1989) showed that neither shading, nor texture, nor motion (or multiple views) gives rise to precise judgments about depth, although all three allow quite precise judgments about the order in depth. It has been concluded that poor precision of depth judgments is not consistent with the claim that vision provides information about metric structure of the 3-D space. As an alternative, Todd and colleagues suggested, on the basis of their results on kinetic depth, that vision can only provide information about the affine structure of 3-D space. Specifically, they showed that the observer can acquire information about Euclidean structure up to depth scaling on the basis of two views. (See also Koenderink \& van Doorn's, 1991, theoretical analysis, which shows that two views determine Euclidean structure up to depth scaling and shear.)

Now, consider accuracy of judgments about depth on the basis of binocular disparity. Foley (1972) showed that depth is not accurately perceived-specifically, that it is underestimated by human observers. Similarly, Johnston (1991) showed that binocular vision provides inaccurate depth information: depth is overestimated for short distances from the observer and underestimated for long distances. Such poor accuracy in binocular depth judgments again implies that vision does not provide information about metric structure of the 3-D space but only information about affine structure, equivalent to Euclidean structure with depth scaling (and possibly with shear).

We are very grateful to Joseph Lappin, Robert Proctor, James Todd, and Peter Werkhoven for comments on an earlier draft of this paper. Correspondence should be addressed to Z. Pizlo, Department of Psychological Sciences, Purdue University, West Lafayette, IN 47907-1364.
In contrast to these studies, Lappin and Love (1992) demonstrated recently, in a set of experiments on depth perception from planar motion, that precision of judgments about distance on a plane slanted in depth is very high (the Weber fraction is less than 3\%). This result seemingly implied that when the constraint of planar motion in 3-D space is imposed, the visual space is metric. We will show, however, by analysis of Lappin and Love's stimuli and results, that high precision in their experiments does not necessarily bear on depth discrimination and, hence, on the structure of the 3-D visual space.

Consider the stimuli first. In Lappin and Love's (1992) experiments, the subject was shown an ellipse represented by a number of points (10 or 20 ) that were placed along the circumference. The ellipse was slanted in depth around the horizontal axis by an angle $\sigma\left(40^{\circ}, 50^{\circ}\right.$, or $\left.60^{\circ}\right)$, whose value varied randomly from trial to trial. In most experimental conditions, the ellipse was oscillating around an axis that was orthogonal to the plane $\pi$ of the ellipse, and this axis did not coincide with the center of the ellipse (in their Experiment 3, the orthogonality restriction was released). This means that the motion of the ellipse was planar and that it consisted of both rotational and translational components: clockwise rotation was associated with translation to the right, and counterclockwise rotation was associated with translation to the left. Lappin and Love hypothesized that because it is computationally easy to reconstruct depth from planar motion (Hoffman \& Flinchbaugh, 1982), it can also be easy perceptually. Three ellipses were used, with ratios of axes (aspect ratio $b / a$ ) equal to $1.06,1.00$, and 0.94 . On each trial one ellipse was shown, and the subject was required to identify the ellipse. Note that the shape (i.e., the aspect ratio) of the retinal image varied randomly from trial to trial and was determined by two factors: the shape (aspect ratio $b / a$ ) of the ellipse and slant $\sigma$. As Lappin and Love correctly pointed out, the effect on the retinal shape of $\sigma$ was greater than the effect of the aspect ratio of the ellipse. This means that in Lappin and Love's experiments, when the ellipse was stationary, the retinal image was not sufficient for subjects to make correct judgments. In fact, in stationary conditions, the subjects' performance was at chance level (except in the case when the slant was zero; i.e., the ellipse was in the frontal plane). However, when the ellipse was rotating, the subjects could discriminate the ellipses above chance level. This result was interpreted by Lappin and Love as representing reliable depth discrimination on a plane slanted in depth.

It has to be pointed out, however, that when a slanted ellipse performs planar oscillatory motion of the kind used by Lappin and Love (1992), the retinal image of the ellipse contains some $2-\mathrm{D}$ cues that could give rise to reliable discrimination without the need of using depth information. Consider Figure 1, which shows perspective images of an ellipse slanted in depth by $\sigma=60^{\circ}$. For 
a

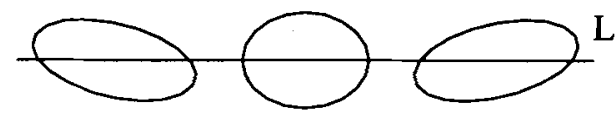

b

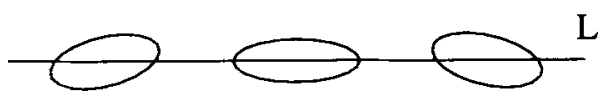

Figure 1. Images of ellipses rotated around the horizontal axis by $\sigma=60^{\circ}$. The aspect ratios of the ellipses are (a) 1.5 and (b) .67 . The ellipses in each row are differently oriented in the plane of the ellipse (see text for more details).

the clarity of our illustration, we used in Figure 1 ellipses with aspect ratios of 1.5 (Figure 1a) and .67 (Figure 1b), which are much more different from 1.00 than the aspect ratios used by Lappin and Love (note that the use of such aspect ratios in our example does not limit the generality of our considerations). Figure 1 shows three views from the sequence used by Lappin and Love. Specifically, on the left-hand side of Figures $1 \mathrm{a}$ and $1 \mathrm{~b}$, the axis $b$ of the ellipse is rotated in the plane $\pi$ by an angle $\phi=45^{\circ}$ counterclockwise from vertical. In the center of these figures, the axis $b$ is vertical $\left(\phi=0^{\circ}\right)$, and on the righthand side the axis $b$ is rotated by $\phi=45^{\circ}$ clockwise from vertical. Denote the intersection of plane $\pi$ with the frontal plane by $L . L$ is a horizontal line going through the center of the retinal image of the ellipse, and this line is in the frontal plane (see Figure 1). This line will be used now to define two cues that were present in the retinal image. One is the width $d$ of the retinal image of the ellipse, measured in the direction of $L$ (i.e., it is the distance between points of intersection of the ellipse and the line $L$ ). The other is the angle $\delta$ between the retinal image of $L$ and the long axis of the retinal image of the ellipse. It has to be pointed out that the line $L$ was not explicitly given in Lappin and Love's stimuli, and, therefore, that the cues $d$ and $\delta$ were probably less noticeable than is implied in Figure 1.

Consider first the width $d$. When the ellipse is rotating within $\pi$, the width $d$ is changing, and these changes do not depend on slant but do depend on the aspect ratio of the ellipse. Namely, if $(b / a)>1$ (as in Figure 1a), $d$ is maximal when $\phi$ is maximal (i.e., the left- and right-hand sides of Figure 1a) and $d$ is minimal when $\phi=0^{\circ}$ (the center). This means that when $\phi$ changes from $45^{\circ}$ counterclockwise through $0^{\circ}$ to $45^{\circ}$ clockwise, $d$ first decreases and then increases. Exactly the opposite happens in the case of an ellipse for which $(b / a)<1$ (see Figure $1 b$ ). Finally, $d$ is constant when the aspect ratio of the ellipse is 1.00 (i.e., the stimulus is a circle). The re- lation between $d$ and $\phi$ is represented by the following formula (see Appendix A for the derivation):

$$
d=2 a /\left[\cos ^{2} \phi+(a / b)^{2} \cdot \sin ^{2} \phi\right]^{1 / 2} .
$$

It is, therefore, clear that the change of the distance $d$ is a cue that could be used to solve Lappin and Love's (1992) task. Recall that $d$ is defined in the frontal plane and that it therefore has nothing to do with the perceived depth either in an unconstrained 3-D space or on a plane slanted in depth. For rotations of the ellipse over the range of $\pm 45^{\circ}, d$ was changing in Lappin and Love's experiment over the range of $3 \%$. This range of changes is likely to give rise to reliable discrimination (see De Valois, Lakshminarayanan, Nygaard, Schlussel, \& Sladky, 1990, and Watt, 1987, who showed that the Weber fraction in discrimination of line lengths on the frontal plane is about $2 \%$ ).

Consider now the angle $\delta$. It can be seen in Figure 1a [i.e., for $(b / a)>1$ ] that when the ellipse is rotated from $\phi=0^{\circ}$ to $\phi=45^{\circ}$ counterclockwise, the orientation $\delta$ of the long axis of the retinal image changes from horizontal (center) to negative. When the same ellipse is rotated from $\phi=0^{\circ}$ to $45^{\circ}$ clockwise, the orientation $\delta$ changes from zero to positive (formulas describing these changes are given in Appendix B). Exactly the opposite is true for the ellipse for which $(b / a)<1$ (Figure $1 b$ ). Obviously, when $(b / a)=1$, the long axis of the retinal image is always horizontal. ${ }^{1}$ In Lappin and Love's (1992) experiment, $\delta$ was changing over the range of several degrees, which seems to be sufficient for discrimination (see Orban, Vandenbussche, \& Vogels, 1984, and Watt, 1987, who showed that threshold in orientation discrimination on the frontal plane is about $1^{\circ}$ ). Thus, in the experiments, the correct responses could be based on discriminating the sign of $\delta$ because this sign is determined exclusively by the aspect ratio; it does not depend on slant. Note, however, that the magnitude of $\delta$ is affected not only by the aspect ratio, but also by slant. In Lappin and Love's experiments, when $\sigma=40^{\circ}$, the amplitude of $\delta$ was $6^{\circ}$, whereas when $\sigma=60^{\circ}$, the amplitude of $\delta$ was $2^{\circ}$. Therefore, the magnitude of $\delta$ confounds the effect of the aspect ratio and slant, which implies that $\delta$ might not be a perfect cue in Lappin and Love's experiments. However, perceptually, the effect of slant on $\delta$ was probably not very strong: When slant is larger, $\delta$ is smaller, but, at the same time, the retinal shape is more elongated and therefore this smaller $\delta$ can still be easily discriminated (see Figure 2 for an illustration of this point, and Jiang, Levi, \& Shah, 1993, for results of psychophysical experiments). Thus, we can conclude that the angle $\delta$ could provide a basis for reliable discrimination in Lappin and Love's experiments.

Our analysis of Lappin and Love's (1992) stimuli showed that using the change of $d$ or the change of $\delta$ (or both) as a cue could transform the original task of judging 3-D metric property (i.e., the aspect ratio of the ellipse on a slanted plane) into the task of judging 2-D ordinal property (i.e., the direction of the changing distance 


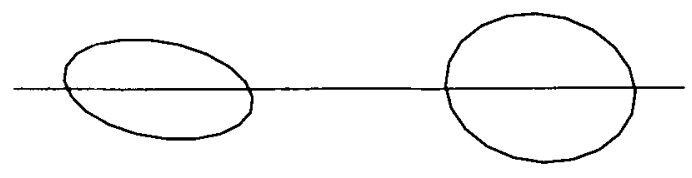

Figure 2. The orientation $\delta$ of the long axis in relation to the horizontal direction in each of the two ellipses is the same and equal to $-10^{\circ}$. It seems easier to discriminate this angle from zero in the case of the more elongated ellipse (the ellipse on the left).

or angle in the retinal image). Therefore, reliable discrimination in Lappin and Love's experiment does not necessarily bear on metric properties of 3-D visual space. The observation that Lappin and Love made in their General Discussion section-namely, that the precision in their task (3-D task) was remarkable and similar to the precision in 2-D tasks in the frontal plane reported by others-is open to an alternative interpretation. In view of our analysis, this similarity of results may not be remarkable: Lappin and Love's " $3-D$ " task could have been a 2-D task in the frontal plane.

We will now analyze the results of Lappin and Love's (1992) experiments in some more detail. We have already discussed their main result-namely, that the precision of judgments was very high-and we have pointed out that this high precision could be based on 2-D cues present in the retinal image, rather than on reliable discrimination of depth. Now, we will show how these 2-D cues can explain other results of Lappin and Love's experiments.

In their first experiment, Lappin and Love (1992) found that the subjects' performance was determined primarily by the aspect ratio of the ellipse and was relatively insensitive to slant (in 1 out of 5 subjects, the effect of slant was equal to the effect of aspect ratio). This result seemed to support the claim that the subjects reliably judged distances on a plane slanted in depth. Note, however, that this result can also be explained by assuming that the subjects were discriminating 2-D cues like $d$ and $\delta$. As pointed out earlier in this note, the magnitude of $d$ is determined only by the aspect ratio, not by the slant of the ellipse. Similarly, the sign of $\delta$ is determined only by the aspect ratio, and the magnitude of $\delta$ is not strongly affected by slant.

In their second experiment, Lappin and Love (1992) showed that when the amplitude of oscillations decreases, precision decreases as well. Lappin and Love suggested that the smaller range of motion reduces visible information, which may lead to worse performance. Note that the result of this experiment can also be explained by the assumption that the subject uses changes of $d$ or of $\delta$ as the cue: for a smaller range of oscillations, the range of changes of $d$ and of $\delta$ is smaller, and, hence, discrimination performance should be worse.

In the third experiment, Lappin and Love (1992) used rotations of the ellipses around an axis that was not orthogonal to plane $\pi$. This arrangement was used in order to test the role of planarity of motion (the motion is pla- nar only when the rotation axis is orthogonal to $\pi$ ). Namely, for nonplanar motion the problem of reconstructing depth is computationally more difficult, and, therefore, it can also be perceptually more difficult. Experimental results confirmed this prediction. Note, however, that the explanation that we propose, which is based on discriminating the change of $d$ or $\delta$, gives the same prediction. Namely, when the axis of rotation is not orthogonal to $\pi$, the derivations presented in Appendices $A$ and $B$ do not hold, and, as a result, there is no longer any simple cue in the retinal image that could be used to discriminate the stimuli. Thus, when the departure of the axis from orthogonality with respect to $\pi$ is larger, the discrimination performance should be worse.

Finally, Lappin and Love (1992) showed that binocular disparity had no effect on the subject's performance. This result implied that even if visual space is metric on a slanted plane, it is not metric in the general case of 3-D space. However, because perception of 2-D features is not likely to benefit from the presence of depth cues, this result is simpler to explain if one assumes, as we do, that the subjects based their judgments on 2-D features present in the retinal image.

This review of Lappin and Love's (1992) experiments shows that their results can be explained equally well or even better by assuming that the subjects' judgments were not based on depth or distance on a slanted plane but, instead, on simple 2-D cues present in the retinal image. Moreover, this new explanation removes the apparent inconsistency between Lappin and Love's results and prior results on depth perception, which showed that perception of distances in depth or on surfaces is imprecise and inaccurate. It has to be pointed out that some of the prior work-namely, that of Lappin and Fuqua (1983)seemingly provided support for Lappin and Love's claims. Lappin and Fuqua showed that subjects could very reliably judge whether a point bisected a segment when the segment was rotating on a plane slanted in depth. Note, however, that bisection of a line segment (or more generally, a comparison of lengths of parallel line segments) is an affine, rather than a metric, property (see Koenderink and van Doorn, 1991, for a treatment of affine and metric properties). Therefore, Lappin and Fuqua's results do not imply that the visual space is metric.

Prior literature provides other examples of experiments that seemingly revealed high precision in 3-D perception tasks involving stimuli on a plane slanted in depth. Stevens and Brookes (1987), Zimmerman and Legge (1989), and Zimmerman and Cavanagh (1990) reported remarkably good precision and accuracy of judgments about slant on the basis of texture. These reports contradicted prior results in which slant judgments were neither precise nor accurate (for a review, see Braunstein, 1976). However, the stimuli in those three reports contained 2-D cues that could be used by subjects. Namely, in all the experiments, the subject was shown a perspective image of texture (e.g., a regular square grid) and of a target (e.g., an ellipse). The task was to adjust the aspect ratio of the target so 
that it represented the same slant as the slant of the texture. Note, however, that this task can be solved by matching the aspect ratio of the retinal image of the texture element with the aspect ratio of the retinal image of the target. Such matching can be very precise and accurate, but it may have nothing to do with slant.

To summarize, there exists substantial experimental evidence implying that vision does not provide metric information about 3-D space or about surfaces in 3-D space. Results that are not consistent with this implication could all have been based on perception of 2-D cues present in the retinal image. Such 2-D cues can give rise to very good performance, but this does not necessarily represent reliable perception of metric properties in 3-D space. It has to be pointed out, however, that our claim that "reliable and accurate judgments about 3-D metric properties are based on 2-D cues present in the retinal image" does not imply that "2-D cues on the retina are always judged reliably and accurately." In fact it is well known that the latter claim is not true. ${ }^{2}$ The difference between these two claims corresponds to the difference between a necessary and a sufficient condition. Namely, the latter claim, which is not true, states that the presence of 2-D cues on the retina is a sufficient condition for reliable and accurate judgments about these cues, whereas the former claim, representing our position, states that the presence of 2-D cues on the retina is a necessary condition for reliable and accurate judgments about metric properties.

These prior results agree with the results of our recent experiments on the discrimination of 3-D shapes on the basis of shading, texture, and contour when only one image of a given shape was presented to the subject (SalachGolyska, Pizlo, Vyain, \& Standish, 1993). Namely, we showed that (1) the precision in the discriminating aspect ratio of an elliptical cylinder viewed from a side is quite poor (the Weber fraction equals $15 \%-50 \%$ ), and (2) the precision in the discriminating aspect ratio of a cuboid is quite good (the Weber fraction is less than $5 \%$ ). This difference in performance between cylinder and cuboid is related to the fact that in the case of a cuboid the subject can use contour-based perspective invariants that do not involve acquiring metric information about 3-D space (Pizlo \& Rosenfeld, 1992). In the case of the cylinder, however, metric information about 3-D space is necessary for discriminating shapes.

We can, therefore, conclude that existing experimental results related to several aspects of 3-D vision (i.e., motion, distance, shape) show that the precision and accuracy of judgments that do not require metric information about 3-D space can be high, but that the precision and accuracy of judgments that do require metric information about 3-D space (or about a surface in 3-D space) are poor. This poor performance implies that the structure of the 3-D visual space is not likely to be metric.

\section{REFERENCES}

Braunstein, M. L. (1976). Depth perception through motion. New York: Academic Press.
De Valois, K. K., Lakshminarayanan, V., Nygaard, R., SchlusSEL, S., \& SLADKY, J. (1990). Discrimination of relative spatial position. Vision Research, 30, 1649-1660.

FolEY, J. M. (1972). The size-distance relation and intrinsic geometry of visual space: Implications for processing. Vision Research, 12, 323-332.

Haralick, R. M., \& Shapiro, L. G. (1992). Computer and robot vision (Vol. 1). New York: Addison-Wesley.

Hochrerg, J. (1971). Perception. In J. W. Kling \& L. A. Riggs (Eds.), Experimental psychology (pp. 475-550). New York: Holt, Rinehart \& Winston.

Hoffman, D. D., \& Flinchbaugh, B. E. (1982). The interpretation of biological motion. Biological Cybernetics, 42, 195-204.

JiANG, B. C., LEVI, D. M., \& SHAH, A. (1993). Spatial-inclination discrimination in two dimensions. Investigative Ophthalmology \& Visual Science, 34, 786. (Abstract)

JoHNSTON, E. B. (1991). Systematic distortions of shape from stereopsis. Vision Research, 31, 1351-1360.

KoEnderink, J. J., \& VAN DOORN, A. J. (1991). Affine structure from motion. Journal of the Optical Society of America A, 8, 377-385.

LAPPIN, J. S., \& FUQUA, M. A. (1983). Accurate visual measurement of three-dimensional moving patterns. Science, 221, 480-482.

LaPPIN, J. S., \& Love, S. R. (1992). Planar motion permits perception of metric structure in stereopsis. Perception \& Psychophysics, 51, 86-102.

Orban, G. A., Vandenbussche, E., \& Vogels, R. (1984). Human orientation discrimination tested with long stimuli. Vision Research, 24, 121-128.

PIZLo, Z., \& Rosenfeld, A. (1992). Recognition of planar shapes from perspective images using contour-based invariants. CVGIP: Image Understanding, 56, 330-350.

Salach-Golyska, M., Pizlo, Z., Vyain, S., \& Standish, J. (1993). 3D shape perception. Investigative Ophthalmology \& Visual Science, 34, 1083. (Abstract)

Stevens, K. A., \& Brookes, A. (1987). Probing depth in monocular images. Biological Cybernetics, 56, 355-366.

Todd, J. T., \& Bressan, P. (1990). The perception of 3-dimensional affine structure from minimal apparent motion sequences. Perception \& Psychophysics, 48, 419-430.

TODD, J. T., \& NoRMaN, J. F. (1991). The visual perception of smoothly curved surfaces from minimal apparent motion sequences. Perception \& Psychophysics, 50, 509-523.

TodD, J. T., \& Reichel, F. D. (1989). Ordinal structure in the visual perception and cognition of smoothly curved surfaces. Psychological Review, 96, 643-657.

WATT, R. J. (1987). Scanning from coarse to fine spatial scales in the human visual system after the onset of a stimulus. Journal of the $O p$ tical Society of America A, 4, 2006-2021.

Zimmerman, G. L., \& Cavanagh, P. (1990). Slant perception at equiluminance. Investigative Ophthalmology \& Visual Science, 31, (Suppl.), 411.

ZIMMERMAN, G. L., \& LEGGE, G. E. (1989). Visual computation of slant using monocular depth cues. Investigative Ophthalmology \& Visual Science, 30 (Suppl.), 263.

\section{NOTES}

1. It has to be pointed out that both $d$ and $\delta$ can be useful only when the subject knows the phase $\phi$ of the motion of the ellipse, because changing the aspect ratio of the ellipse from greater than one to less than one (or vice versa) has the same effect on $d$ and $\delta$ as changing the phase $\phi$. It is clear, however, that in Lappin and Love's (1992) experiments, subjects knew the phase $\phi$ because this phase was easy to detect from the translational component of the motion of the ellipse.

2. For example, the subject cannot accurately judge the size of the retinal image when depth cues are present. Specifically, if perceived distance from a stimulus changes, but the retinal size is constant, the perceived size of the stimulus is not constant, but instead changes in the direction predictable from depth cues (see Hochberg, 1971, pp. 507-510, for a review of size perception). 


\section{APPENDIX A}

Let $a$ be the horizontal semiaxis of an ellipse and $b$ its vertical semiaxis. Assume, without restricting generality, that the center of the ellipse coincides with the origin of the $(x, y)$ coordinate system. This ellipse is represented by

$$
x^{2} / a^{2}+y^{2} / b^{2}=1 .
$$

Now, we rotate this ellipse by an angle $\phi$, and we measure the width $\boldsymbol{d}$ of the ellipse along the $x$-axis. Note that instead of rotating the ellipse, one can rotate the horizontal line $y=0$ by an angle $-\phi$ and then measure the width of the ellipse along this line. After rotation, this line intersects the ellipse at two points:

$$
\begin{array}{ll}
x_{1}=+d / 2 \cdot \cos \phi ; & y_{1}=-d / 2 \cdot \sin \phi \\
x_{2}=-d / 2 \cdot \cos \phi ; & y_{2}=+d / 2 \cdot \sin \phi .
\end{array}
$$

We now substitute $x$ and $y$ in (A1) by (A2) and solve for $d$ :

$$
d=2 a /\left[\cos ^{2} \phi+(a / b)^{2} \cdot \sin ^{2} \phi\right]^{1 / 2} .
$$

\section{APPENDIX B}

Let us rotate the ellipse (A1) by an angle $\phi$ around the origin of the $(x, y)$ coordinate system:

$(x \cos \phi+y \sin \phi)^{2} / a^{2}+(-x \sin \phi+y \cos \phi)^{2} / b^{2}=1$.

Now, we determine an orthographic image of the ellipse (B1) assuming that this ellipse was rotated in depth around the $x$-axis with slant $\sigma$ (we use orthographic transformation rather than perspective, because orthographic transformation is computationally much easier and, at the same time, it is a good approximation to perspective for such small stimuli as those used by Lappin and Love (1992) (about $1^{\circ}$ of arc):

$$
\begin{aligned}
& x^{2} \cdot\left(b^{2} \cos ^{2} \phi+a^{2} \sin ^{2} \phi\right) /(a b)^{2} \\
& +y^{2} \cdot\left(b^{2} \sin ^{2} \phi+a^{2} \cos ^{2} \phi\right) /(a b \cos \sigma)^{2} \\
& +2 x y \sin \phi \cos \phi\left(b^{2}-a^{2}\right) /\left[(a b)^{2} \cos \sigma\right]=1 .
\end{aligned}
$$

Let $\mathbf{v}=(x, y)$ be a column vector representing a point in $(x, y)$ coordinate system. Then, Equation B2 can be rewritten in matrix notation as

$$
\begin{aligned}
& \qquad \begin{aligned}
\mathbf{v}^{\mathbf{T}} \mathbf{A v} & =1, \\
\text { where } \mathbf{A} & =\left[\begin{array}{ll}
d & e \\
e & f
\end{array}\right] \\
\text { and } d & =\left(b^{2} \cos ^{2} \phi+a^{2} \sin ^{2} \phi\right) /(a b)^{2} \\
e & =\sin \phi \cos \phi\left(b^{2}-a^{2}\right) /\left[(a b)^{2} \cos \sigma\right] \\
f & =\left(b^{2} \sin ^{2} \phi+a^{2} \cos ^{2} \phi\right) /(a b \cos \sigma)^{2} .
\end{aligned}
\end{aligned}
$$

The orientation $\delta$ of the long axis of the ellipse (B3) can be found through determining eigenvalues and eigenvectors of $\mathbf{A}$ (Haralick \& Shapiro, 1992):

$\tan \delta=2 e /\left\{f-d+\left[(f-d)^{2}+4 e^{2}\right]^{1 / 2}\right\}$.

(Manuscript received March 8, 1993; revision accepted for publication June 18, 1993.) 